\title{
Simulation of Laser-Accelerated Proton Focusing and Diagnosis with a Permanent Magnet Quadrupole Triplet
}

\author{
Hironao SAKAKI，Mamiko NISHIUCHI，Toshihiko HORI，Paul R. BOLTON，Motonobu TAMPO, \\ Akifumi YOGO, Kiminori KONDO, Shunichi KAWANISHI, Hiroshi IWASE ${ }^{1)}$ and Koji NIITA ${ }^{2)}$ \\ Japan Atmic Energy Agency, 8-1-7 Umemidai, Kizugawa, Kyoto 619-0215, Japan \\ ${ }^{1)}$ High Energy Accelerator Research Organization, KEK, 1-1, Oho, Tsukuba, Ibaraki 305-0801, Japan \\ ${ }^{2)}$ Research Organization for Information Science and Technology, RIST, 2-4, Shirakata, Tokai, Ibaraki 319-1106, Japan
}

(Received 10 January 2010 / Accepted 19 January 2010)

\begin{abstract}
The Monte Carlo Particle and Heavy Ion Transport code (PHITS) was used for diagnosing the focusing and defocusing action of a triplet of permanent magnet quadrupoles on laser-accelerated proton bunches of short duration. The code predicts reasonably well the observed transverse beam profile as measured with a CR-39 track detector demonstrating that PHITS can be used to simulate proton bunch transport by conventional ion optics in the laser-driven case.
\end{abstract}

(c) 2010 The Japan Society of Plasma Science and Nuclear Fusion Research

Keywords: laser-accelerated ion, transverse profile, CR-39, high energy hadron transport code

DOI: $10.1585 /$ pfr.5.009

Ongoing studies of laser-acceleration of ions can be pursued with many applications in such as the development of more compact laser-driven medical accelerators. The generation of energetic protons and ions from laserplasma interactions, has made not only fast ignition in ICF but also laser-driven ion radiotherapy (L-IBRT) [1] a subject of strong interest.

Applications to L-IBRT can have stringent requirements for beam monitoring and control to guarantee highly localized and safe irradiation of affected tissue. In previously published work the beam focusing action of a permanent magnet quadrupole (PMQ) doublet was demonstrated [2]. It is therefore important to examine proton beam transport with more conventional optics and to evaluate the predictive capability of simulation codes in the laser-driven case with such optics configurations.

We report here the demonstrated low energy proton focusing by a PMQ triplet and the use of solid track detector film (CR-39) to measure the transverse spatial beam profiles. Simulated transverse profiles of energy deposition in CR-39 film by the PHITS (Particle and Heavy Ion Transport code system) code [3] are shown to be in reasonably good agreement with observed results.

The PMQ triplet was aligned with the beamline intersecting the proton source and normal to its surface. The J-KAREN laser system at the Kansai Photon Science Institute (KPSI) of JAEA provided P-polarized laser irradiation at a $45^{\circ}$ incident angle with pulses of central wavelength $800 \mathrm{~nm}$, duration of 45 femtoseconds (FWHM), and energy of $630 \mathrm{~mJ}$ at a $1 \mathrm{~Hz}$ repetition rate.

The laser target was a polyimide tape of $12 \mu \mathrm{m}$ thick-

author's e-mail: sakaki.hironao@jaea.go.jp ness. Peak irradiances up to $10^{19} \mathrm{~W} / \mathrm{cm}^{2}$ were achieved on target and the intensity contrast ratio was set to $10^{-10}$ level.

A sketch of the PMQ configuration is seen in Fig. 1. The first PMQ is of field strength (length) $55 \mathrm{~T} / \mathrm{m}(50 \mathrm{~mm})$ and located $79.5 \pm 0.5 \mathrm{~mm}$ downstream of the laser irradiation point. The second PMQ field strength (length) is $-40 \mathrm{~T} / \mathrm{m}(50 \mathrm{~mm})$ and located $128.5 \pm 0.5 \mathrm{~mm}$ downstream of the first PMQ. The third PMQ field strength (length) is $60 \mathrm{~T} / \mathrm{m}(20 \mathrm{~mm})$ and located $89.3 \pm 0.5 \mathrm{~mm}$ downstream from the second PMQ. As is also shown in Fig. 1 the CR39 detector (with an upstream aluminum range moderator of $12.5 \mu \mathrm{m}$ thickness) was placed downstream of the proton source along the beamline at distances ranging from 450 to $850 \mathrm{~mm}$ downstream.

Figure 2 reveals the transverse beam profile obtained from CR-39 following a 20 minute etching process in a $70^{\circ} \mathrm{C} 7 \mathrm{~N} \mathrm{KOH}$ solution. It shows the proton focusing and orthogonal defocusing and by the PMQ triplet. The etching rate in this case was $1.3 \mu \mathrm{m} /$ hour so a 20 minutes process etches to $0.45 \mu \mathrm{m}$ indicating the number of protons at this depth. The aluminum filter thickness suggests that the proton energy incident on the CR-39 exceeds $0.9 \mathrm{MeV}$. The number of protons reaching CR-39 at the $850 \mathrm{~mm}$ downstream distance was counted. Upstream (distances from 450 to $750 \mathrm{~mm}$ from the target) the proton bunch density at the core exceeded the spatial resolution capability of CR39 , rendering the image core transparent as seen in Fig. 2.

Figure 3 (a) shows CR-39 profile at the $850 \mathrm{~mm}$ location and the magnified pit images obtained with a microscope. The average pit areal density (seen as white in the figure) is $2.2 \times 10^{2} / 100 \mu \mathrm{m}^{2}$. Considering the whole white pitted area, we estimate about $2.9 \times 10^{8}$ protons in CR-39 at this location. 


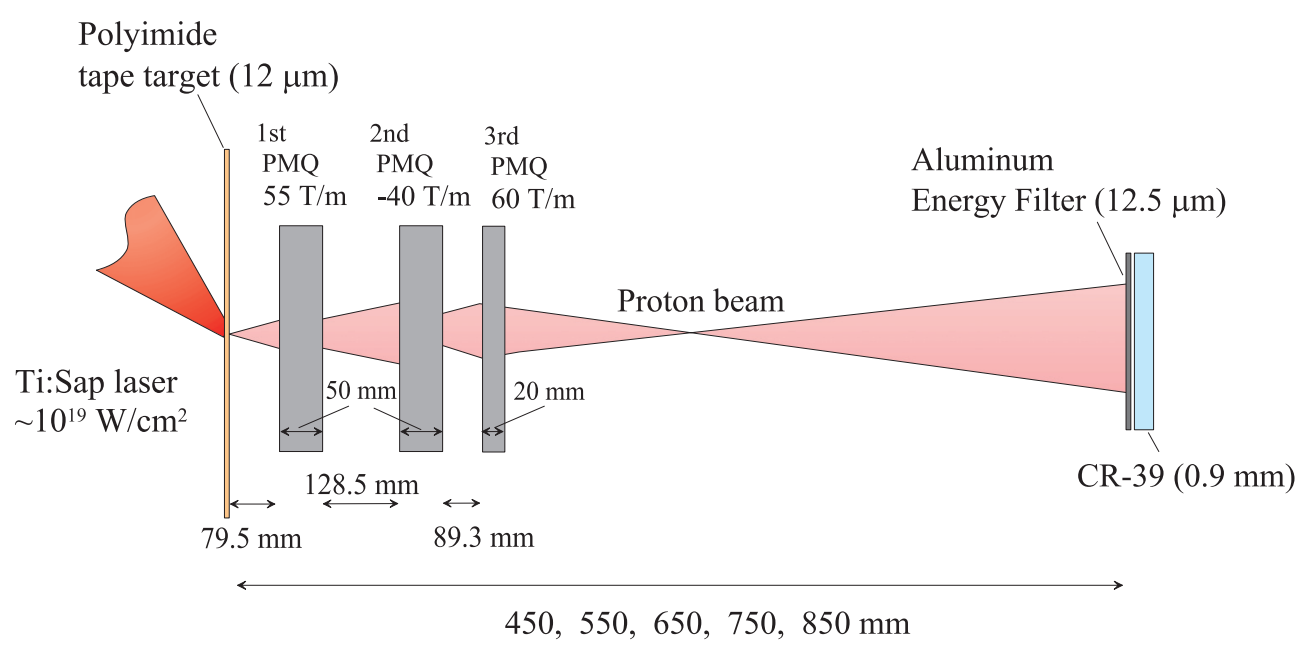

Fig. 1 Experimental configuration with the ermanent magnet quadrupole triplet and the CR-39 detector.
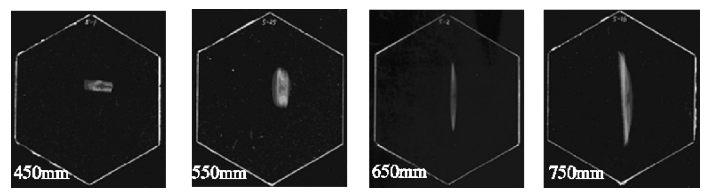

Fig. 2 Focusing and defocusing results from CR-39 measurements at locations 450 to $750 \mathrm{~mm}$ downstream of the proton source.
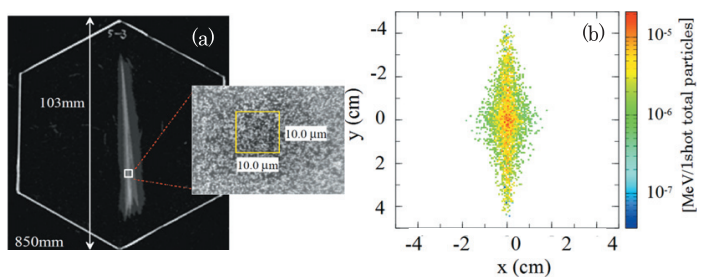

Fig. 3 (a) Transverse bunch profile at $850 \mathrm{~mm}$ obtained with CR-39. The surface was magnified with a microscope such that the number of pits (for a $100 \mu \mathrm{m}^{2}$ area) could be counted. (b) PHITS simulation of energy deposit in CR$39850 \mathrm{~mm}$ from the source relevant to a $0.45 \mu \mathrm{m}$ depth.

We examined the energy deposit in the CR-39 at $850 \mathrm{~mm}$ using PHITS which incorporates the quantum molecular dynamics model for simulating nucleus-nucleus interactions. In addition to the energy deposit in CR-39, PHITS also simulates the focusing/defocusing action of the PMQ elements.

The simulated transverse profiles to the $0.45 \mu \mathrm{m}$ depth are illustrated in Fig. 3 (b) using a pseudocolour scale that is in units of deposited energy per 'source' proton per laser shot $[\mathrm{MeV} / 1$ shot total particles]. The intersection with the beam line is at the origin, $x=0, y=0$. The normalization factor is therefore the total proton number (full spectrum) at the source point where we also assume for simplicity zero bunch duration at birth. The proton energy distribution at the source has a $3 \mathrm{MeV}$ cutoff, an energy spread near $100 \%$ and a divergent half angle of $\sim 10^{\circ}$. These are the input parameters used by PHITS to simulate the CR-39 transverse profile with 50,000 protons. The PHITS result also shows that this profile was formed by the $25.3 \%$ of the total number of protons accelerated by a single laser pulse.

As reported elsewhere the total number of protons has been independently measured by time-of-flight (TOF) spectrometry [4] to be $\sim 10^{9}$. Therefore, the simulated proton number on CR-39 is about $2.5 \times 10^{8}$ which coincides well with the experimentally obtained result.

In conclusion, the general-purpose Monte Carlo code, PHITS was used to simulate both focusing/defocusing PMQ and the associated transverse spatial profile established on a CR-39 film detector. Agreement with observed data is good. This is an all-in-one code which simulates from the interaction of quadrupole magnetic fields to the CR-39's etching image concurrently. Consequently PHITS can be used to simulate the proton transport in the laser-driven case. These developments represent steps of progress toward the development of an integrated laserdriven ion accelerator system (ILDIAS).

This work is supported by Special Coordination Found (SCF) for Promoting Science and Technology commissioned by the Ministry of Education, Culture, Sports, Science and Technology (MEXT) of Japan.

[1] S.V. Bulanov et al., Plasma Physics Reports 28-5, 453, (2002).

[2] M. Nishiuchi et al., Appl. Phys. Lett. 94, 061107 (2009).

[3] K. Niita et al., Radiat. Meas. 41, 1080 (2006).

[4] A. Yogo et al., Phys. Plasmas 14, 043104 (2007). 